TRANSACTIONS OF THE

AMERICAN MATHEMATICAL SOCIETY

Volume 188, Issue 2, 1974

\title{
UNIFORMLY DISTRIBUTED SEQUENCES IN LOCALLY COMPACT GROUPS. I(1)
}

BY

\section{LEONORA BENZINGER}

ABSTRACT. We investigate the notion of uniformly distributed sequences in locally compact groups. Our main result is the following: A locally compact group $G$ possesses a uniformly distributed sequence if and only if it possesses a sequence whose homomorphic images are dense in each of the compact quotients of G.

Introduction. H. Weyl [10] introduced and studied the notion of a uniformly distributed sequence on the closed interval $[0,1]$. This notion was extended to compact groups and investigated by Eckmann [3]. Rubel [9] extended this idea to locally compact groups. Under Rubel's definition, a sequence $\left\{g_{v}\right\}$ is uniformly distributed in a locally compact group $G$ if its homomorphic images are uniformly distributed in all compact quotients of $G$. By a compact quotient we mean a compact quotient of the form $G / H$ where $H$ is a closed normal subgroup of $G$. We say that $H$ is of compact index in $G$, see [9]. We denote by $\phi_{H}$ the natural homomorphism mapping $G$ onto $G / H$.

"Uniformly distributed" will be abbreviated u.d.

Other definitions for a u.d. sequence are possible. For example, S. Hartman [5] introduced a notion of a u.d. sequence on noncompact groups which is not equivalent to Rubel's definition. For a comparison of these two definitions on locally compact abelian groups see Berg, Rajagopalan, and Rubel [1, p. 443].

We say a locally compact group $G$ is $K$-separable if it possesses a sequence $\left\{x_{v}\right\}$ whose homomorphic images are dense in each of the compact quotients of $G$, compare with $[1, \mathrm{p} .437]$. We say that the sequence $\left\{x_{v}\right\}$ is $K$-dense in $G$, compare with [1, p. 441].

This paper contains a generalization to locally compact groups of some results concerning uniformly distributed sequences on locally compact abelian groups of Berg, Rajagopalan, and Rubel [1]. In the first section we present some

Presented to the Society, January 18, 1972 under the title Uniformly distributed sequences on locally compact groups; received by the editors February 13, 1973.

AMS (MOS) subject classifications (1970). Primary 22D05; Secondary $10 \mathrm{~K} 99$.

Key words and phrases. Locally compact group, uniform distribution, finite dimensional unitary representation, compact quotient, periodic representation.

(1) This paper contains material from the author's doctoral dissertation submitted to Syracuse University.

Copyright $\odot$ 1974, American Mathematical Society 
preliminary definitions and theorems and we include discussions of those immediate consequences which are useful for investigating uniformly distributed sequences in locally compact groups. Among those consequences, we have an extension of the Weyl criterion to locally compact groups and a discussion of the periodic representations (those representations which may be considered as representations of compact quotients) of locally compact groups.

The following lemma is fundamental to the proof of our theorem. It is the tool we use to construct uniformly distributed sequences on $K$-separable locally compact groups.

Key lemma. Let $G$ be a locally compact group and $H$ a countable subgroup. Let $S$ be a set of finite dimensional irreducible unitary representations of $G$ and suppose that each nontrivial $\rho$ in $S$ is nontrivial and irreducible as a representation of $H$. Then there exists a sequence $\left\{b_{v}\right\}$ in $H$ sucb that

$$
\lim _{n \rightarrow+\infty} \frac{1}{n} \sum_{\nu=1}^{n} \rho\left(b_{\nu}\right)=0
$$

for all nontrivial $\rho$ in $S$.

The second section contains a discussion of an example which plays a crucial role in the proof of the key lemma and which illustrates some of the difficulties characteristic of nonabelian groups which arise in the proof of this lemma. Because of these difficulties (which basically stem from the fact that we are dealing with finite dimensional representations as opposed to 1-dimensional representations in the abelian case), the proof involves a rather complicated construction of the desired sequence. The proof of the validity of this sequence includes the introduction of the notion of $F$-index of a finite dimensional unitary representation of a locally compact group. The notion of $F$-index is a refinement of the idea of a fixed point free representation which was used by Eckmann [3] in his study of uniformly distributed sequences on compact groups.

The third section contains the completion of the proof of the key lemma. The proof of our main theorem follows as a consequence of the key lemma and the Weyl criterion for locally compact groups.

1. Preliminary definitions and results. We present some basic definitions concerning uniform distributions on compact and locally compact groups.

(1.1) Notation. Let $G$ be a locally compact group, $f$ a complex-valued function on $G$, and $\left\{g_{v}\right\}$ a sequence of elements in $G$. By $\left\langle f,\left\{g_{v}\right\}\right\rangle$ we mean the following limit if it exists:

$$
\left\langle f,\left\{g_{v}\right\}\right\rangle=\lim _{n \rightarrow+\infty} \frac{1}{n} \sum_{\nu=1}^{n} f\left(g_{v}\right) .
$$


(1.2) Definition (Eckmann [3]). If $K$ is a compact group, the sequence $\left\{g_{v}\right\}$ in $K$ is uniformly distributed if for each continuous complex-valued function $f$ on $K,\left\langle f,\left\{g_{v}\right\}\right\rangle=\int_{K} f d \mu$ where $\mu$ is normalized Haar measure on $K$.

(1.3) We recall that a representation of a locally compact group $G$ is a homomorphism from $G$ into the operators on a Hilbert space. A finite dimensional representation $\rho$ of $G$ of dimension $m$ is a homomorphism from $G$ into the operators on an $m$-dimensional Hilbert space. We shall assume all our representations are continuous and refer to them simply as representations. We note that with respect to some basis, each finite dimensional representation $\rho$ of $G$ of dimension $m$ may be considered as the $m \times m$ matrix $\left[\rho_{i j}\right]$ where each $\rho_{i j}$ is a continuous complex-valued function on $G$.

(1.4) Definition. We call each function $\rho_{i j}$ a minimal almost invariant function associated with the finite dimensional representation $\rho$ and we shall use the abbreviation m.a.i.

(1.5) Notation. Let $\left\{g_{v}\right\}$ be a sequence in $G$ and let $\rho$ be a finite dimensional representation of $G$ of dimension $m$. By $\left\langle\rho,\left\{g_{v}\right\}\right\rangle$ we mean the $m \times m$ matrix $\left[\left\langle\rho_{i j},\left\{g_{v}\right\}\right\rangle\right]$ provided $\left\langle\rho_{i j},\left\{g_{v}\right\}\right\rangle$ exists for each $\rho_{i j}$.

(1.6) Theorem (Eckmann [3]). A sequence $\left\{g_{v}\right\}$ is $u_{0} d$. in a compact group $K$ if and only if $\left\langle\rho,\left\{g_{v}\right\}\right\rangle=0$ for eacb nontrivial irreducible representation $\rho$ of $K$.

This theorem is the analogue for compact groups of a necessary and sufficient condition due to $\mathrm{H}$. Weyl [10] that a sequence of real numbers be u.d. in the closed interval $[0,1]$. We shall refer to Theorem 1.6 as the Weyl criterion for compact groups.

(1.7) Definition. A representation $\rho$ of $G$ is periodic if it is constant on the cosets of some closed normal subgroup $H$ of compact index. We then say that $\rho$ is of period $H$, compare with Berg and Rubel $[2, \mathrm{p} .85]$. We observe that the choice of period for $\rho$ is not in general unique.

An irreducible periodic representation $\rho$ of $G$ is finite dimensional. Indeed, we consider $\rho$ as a representation of $G / H$ for some closed normal subgroup $H$ of compact index and define $\rho\left(\phi_{H}(g)\right)=\rho(g)$ for each $g \in G$. If $\rho$ as a representation of $G$ is irreducible then it is irreducible as a representation of $G / H$. But then $\rho$ is an irreducible representation of a compact group and hence must be finite dimensional [6, Theorem 22.13].

Suppose $\rho$ is an irreducible periodic representation of $G$ of period $H$. Since any irreducible representation of a compact group is equivalent to a unitary representation, $\rho$ as a representation of $G / H$ is equivalent to a unitary representation $\sigma$. We extend $\sigma$ to all of $G$ in the natural way. Obviously, $\sigma$ as a representation of $G$ is unitary and equivalent to $\rho$. There is no loss of generality then, if we restrict ourselves to irreducible periodic representations of $G$ which are unitary. 
(1.8) Notation. Let $R(G)$ denote the set of equivalence classes of the finite dimensional irreducible unitary representations of $G$. When no confusion is likely, we shall frequently speak of a representation $\rho$ in $R(G)$. Depending upon the context in which it is used, it will be clear whether we mean a particular representation $\rho$ or the equivalence class which contains $\rho$.

(1.9) Notation. Let $P$ denote the set of periodic irreducible unitary representations of $G$.

As a result of the preceding definitions and discussion we easily obtain the extension of Theorem 1.6 to locally compact groups.

(1.10) Theorem (Weyl criterion for locally compact groups). Let $G$ be a locally compact group. The sequence $\left\{g_{v}\right\}$ is $u_{0} d$. in $G$ if and only if for each nontrivial $\rho \in P,\left\langle\rho,\left\{g_{v}\right\}\right\rangle=0$.

2. An example. Part of the proof of the key lemma depends upon the idea of generating in a locally compact group $G$ a sequence which averages a single representation $\rho \in R(G)$ to 0 from some given sequences in $G$ which average certain m.a.i. functions of $\rho$ to 0 . We now give the construction which we shall use for generating a new sequence from a collection of sequences which are already given. We shall use this general construction once in this section and twice in the next section.

(2.1) Suppose that we have a countable collection of sequences of elements $\{c(1, v)\},\{c(2, v)\}, \cdots,\{c(k, v)\}, \cdots$ in a locally compact group $G$. For the purposes of the present section we may think of $G$ as a nontrivial irreducible group of $m \times$ $m$ unitary matrices. In some cases which follow the collection of distinct sequences will actually be finite. We use these sequences to generate a new sequence $\left\{c_{v}\right\}$ in $G$ which we construct in stages. The first stage is $c(1,1)$. The second stage is $c(2,1) c(1,1), c(2,1) c(1,2), c(2,2) c(1,1), c(2,2) c(1,2)$. The third stage is $c(3,1) c(2,1) c(1,1), c(3,1) c(2,1) c(1,2), c(3,1) c(2,1) c(1,3)$, $c(3,1) c(2,2) c(1,1), c(3,1) c(2,2) c(1,2), c(3,1) c(2,2) c(1,3), c(3,1) c(2,3) c(1,1)$, $\cdots, c(3,1) c(2,3) c(1,3), c(3,2) c(2,1) c(1,1), \ldots, c(3,2) c(2,1) c(1,3), \ldots$, $c(3,2) c(2,3) c(1,1), \ldots, c(3,2) c(2,3) c(1,3), c(3,3) c(2,1) c(1,1), \ldots$, $c(3,3) c(2,3) c(1,3)$. The $n$th stage is all terms $c\left(n, t_{n}\right) c\left(n-1, t_{n-1}\right) \cdots c\left(1, t_{1}\right)$ where $t_{j}$ runs from 1 to $n, j=1,2, \cdots, n$ ordered in a manner which we shall now describe. The subscript $t_{1}$ runs from 1 to $n$ and recycles while the other $t_{j}$ remain constant. The subscript $t_{2}$ runs from 1 to $n$ in blocks of $n$ and then recycles while $t_{3}$ to $t_{n}$ remain constant. In general, $t_{j}$ runs from 1 to $n$ in blocks of $n^{j-1}$ and then (provided $j \neq n$ ) recycles while $t_{j+1}$ through $t_{n}$ remain constant. The ordering of the terms in the $n$th stage is the natural ordering of the numbers $t_{n} t_{n-1} \cdots t_{1}$ written in the base $n$ using as digits the integers 1 through $n$. 
There are $n^{n}$ such numbers. Thus there are $n^{n}$ terms in the $n$th stage of the sequence $\left\{c_{v}\right\}$. Let $C_{n}$ denote the $n$th stage.

The counting function for $\left\{c_{v}\right\}$ is given in terms of an $n$-tuple $\left(t_{n}, t_{n-1}, \cdots\right.$, $\left.t_{1}\right)$ by

$$
v=\sum_{i=1}^{n-1} i^{i}+\sum_{j=2}^{n}\left(t_{j}-1\right) n^{j-1}+t_{1}
$$

however, we find in discussing particular terms of the sequence that it is more convenient to specify the $n$-tuples $\left(t_{n}, t_{n-1}, \cdots, t_{1}\right)$ than to specify the subscripts $v$.

For a fixed positive integer $n$ and any positive integer $q$ it is convenient to partition $C_{n+q}$ into $(n+q)^{q}$ blocks $B(i, q, n)$. We define the blocks $B(i, q, n)$ in terms of the $(n+q)$-tuples $\left(t_{n+q}, t_{n+q-1}, \cdots, t_{1}\right), 1 \leq t_{j} \leq n+q, j=1,2, \cdots, n+q \cdot A$ block $B(i, q, n)$ consists of those members $c\left(n+q, t_{n+q}\right) \cdots c\left(n, t_{n}\right) \cdots c\left(1, t_{1}\right)$ of the $C_{n+q}$ stage for which the indices $t_{n}, \cdots, t_{1}$ cycle from 1 to $n+q$ while the remaining indices are fixed. The ordering within the block is the natural one induced by the ordering in the $C_{n+q}$ stage. In general, $B(i, q, n)$ is

$$
\begin{aligned}
& \left\{c_{v} \in C_{n+q} \mid t_{n+q}, t_{n+q-1}, \cdots, t_{n+1}\right. \text { are constant and } \\
& \left.\qquad i=\sum_{j=2}^{q}\left(t_{n+j}-1\right)(n+q)^{j-1}+t_{n+1}\right\} \text { if } q \geq 2
\end{aligned}
$$

and

$$
\left\{c_{v} \in C_{n+q} \mid t_{n+q}, t_{n+q-1}, \cdots, t_{n+1} \text { are constant and } i=t_{n+1}\right\} \text { if } q=1
$$

with the natural ordering of the $C_{n+q}$ stage. There are $(n+q)^{q}$ different $q$ tuples $\left(t_{n+q}, \cdots, t_{n+1}\right)$. Consequently, $i$ runs from 1 to $(n+q)^{q}$. The increasing order of the blocks $B(i, q, n)$ is the one induced by the natural ordering of the numbers of the form $t_{n+q} t_{n+q-1} \cdots t_{n+1}$ written in the base $n+q$ using as digits the integers 1 through $n+q$. There are $(n+q)^{n}$ terms in each block.

(2.2) Definition. For a fixed integer $n$ we shall call each of the blocks $B(i, q, n)$ an $n t b$ stage-like block.

We now consider an example of a sequence $\left\{c_{v}\right\}$ of elements which we construct according to (2.1) in an irreducible group $G$ of $m \times m$ unitary matrices. The consideration of this example forms a fundamental part of the proof of the key lemma.

(2.3) Notation. If $B$ is a set, by $n(B)$ we mean the number of elements in $B$.

(2.4) Notation. For any sequence $\left\{c_{v}\right\}$ in an irreducible group $K$ of $m \times \cdot m$ 
unitary matrices let $\left\langle\left\{c_{v}\right\}\right\rangle$ denote the following limit provided it exists:

$$
\left\langle\left\{c_{v}\right\}\right\rangle=\lim _{n \rightarrow+\infty} \frac{1}{n} \sum_{\nu=1}^{n} c_{v}
$$

(2.5) Definition. Suppose that $d$ is the $m \times m$ unitary matrix $\left[d_{i j}\right]$. By $\|d\|$ we mean max $\left\|d_{i j}\right\|$.

In Lemma (2.6) (which is actually a result about a bounded sequence of numbers constructed according to (2.1)) we obtain some sufficient conditions which are fairly easy to verify and which guarantee that $\left\langle\left\{c_{v}\right\}\right\rangle=0$. These conditions are that the average of $\left\{c_{v}\right\}$ over all $n$th stages and $n$th stage-like blocks must be suitably small in norm for all $n$ sufficiently large. This lemma is the primary tool we use for proving that the sequences we generate average certain sets of representations to 0 .

(2.6) Lemma. Let $\left\{c_{v}\right\}$ be a sequence in $K$ which is constructed in stages according to (2.1). Suppose that for eacb $\epsilon>0$ there exists $N$ sucb that for all $n>$ $N$ we bave

$$
\begin{gathered}
\left\|\frac{1}{n^{n}} \sum_{c_{v} \in C_{n}} c_{v}\right\|<\epsilon / 4, \\
\left\|\frac{1}{(n+q)^{n}} \sum_{c_{v} \in B(i, q, n)} c_{v}\right\|<\epsilon / 4, \quad 1 \leq i \leq(n+q)^{q}
\end{gathered}
$$

for each positive integer $q$. Then there exists an integer $M$ so that for all $p>M$, $\left\|p^{-1} \sum_{\nu=1}^{p} c_{v}\right\|<\epsilon$.

Proof. Let $n>N$ and fix it. There exists a positive integer $Q_{0}$ so that for all $q>Q_{0}$, the number of terms in an $n$th stage-like block $B(i, q, n)$ divided by the number of terms in the sequence $\left\{c_{v}\right\}$ up to and including those in $B(i, q, n)$ is less that $\epsilon / 4$. Set

$$
M=\max \left\{\sum_{j=1}^{n+Q_{0}} j^{j}, \frac{4}{\epsilon} \sum_{j=1}^{n-1} j^{j}\right\}
$$

Let $p$ be any integer greater than $M$ and let $D=\left\{c_{1}, c_{2}, \cdots, c_{p}\right\}$. We partition $D$ into subsets determined by the stages in the formation of $\left\{c_{v}\right\}$. Since $D$ is finite, there is a stage, say $C_{n+q}$, of which $D \cap C_{n+q}$ is a proper subset but $D \cap C_{j}=C_{j}$ for $j=1,2, \cdots, n+q-1$ and $D \cap C_{j}=\varnothing$ for $j>n+q$. We partition $C_{n+q}$ into blocks $B(i, q, n)$. For some integer $i_{0}, 1 \leq i_{0} \leq(n+q)^{q}, D \cap$ $B\left(i_{0}, q, n\right)$ is a proper subset of $B\left(i_{0}, q, n\right)$. We have 


$$
\begin{aligned}
& \left\|\frac{1}{p} \sum_{\nu=1}^{p} c_{\nu}\right\|=\| \frac{1}{p}\left(\sum_{j=1}^{n-1} \sum_{c_{\nu} \in C_{j}} c_{v}+\sum_{j=n}^{n+q-1} \sum_{c_{v} \in C_{j}} c_{v}\right. \\
& \left.+\sum_{j=1}^{i_{0}} \sum_{c_{v} \in B(j, q, n) \cap D} c_{v}\right) \| \\
& \leq\left\|\frac{1}{p} \sum_{j=1}^{n-1} \sum_{c_{v} \in C_{j}} c_{v}\right\|+\sum_{j=0}^{q-1} \frac{(n+j)^{n+j}}{p} \\
& \cdot\left\|\frac{1}{(n+j)^{n+j}} \sum_{c_{v} \in C_{n+j}} c_{v}\right\| \\
& +\sum_{j=1}^{i_{0}} \frac{(n+q)^{n}}{p}\left\|\frac{1}{(n+q)^{n}} \sum_{c_{v} \in B(j, q, n)} c_{v}\right\| \\
& +\left\|\frac{1}{p} \sum_{c_{v} \in B\left(i_{0}, q, n\right) \cap D} c_{v}\right\| \\
& <\frac{1}{p} \sum_{j=1}^{n-1} j^{j}+\frac{\epsilon}{4} \sum_{j=0}^{q-1} \frac{(n+j)^{n+j}}{p} \\
& +\sum_{j=1}^{i_{0}} \frac{(n+q)^{n}}{p \cdot}\left\|\frac{1}{(n+q)^{n}} \sum_{c_{v} \in B(j, q, n)} c_{v}\right\| \\
& +\left\|\frac{1}{p} \sum_{c_{\nu} \in B\left(i_{0}, q, n\right) \cap D} c_{v}\right\|
\end{aligned}
$$

by (i) and the fact that each matrix $c_{v}$ is unitary. By the choice of $p>M$ and by assumption (ii) we have

$$
\left\|\frac{1}{p} \sum_{v=1}^{p} c_{v}\right\|<\frac{3 \epsilon}{4}+\frac{n\left(B\left(i_{0}, q, n\right)\right)}{p} .
$$

Since $p>M$, it follows that $q>Q_{0}$. Thus

$$
\left\|\frac{1}{p} \sum_{\nu=1}^{p} c_{v}\right\|<\epsilon .
$$


From Lemma (2.6) we have sufficient conditions in terms of averages over $n$th stages and $n$th stage-like blocks for a sequence $\left\{c_{v}\right\}$ in $K$ constructed according to (2.1) to average to 0 . The construction of $\left\{c_{v}\right\}$ is not complete, however, because we have not specified the generating sequences $\{c(1, v)\},\{c(2, v)\}, \ldots,\{c(k, v)\}$, ... for $\left\{c_{v}\right\}$ which will satisfy the hypotheses of Lemma (2.6). The next lemma enables us to obtain these generating sequences for the sequence $\left\{c v_{v}\right\}$ in $K$.

(2.7) Definition. If $a \in K$, then the fixed subspace of $a$ is $\left\{x \in C^{m} \mid a x=x\right\}$ and is denoted by $F_{a}$.

(2.8) Lemma. Let $K$ be an irreducible group of $m \times m$ unitary matrices. Then there exists a finite subset of $K,\left\{a_{1}, a_{2}, \cdots, a_{l}\right\}$ with $1 \leq l \leq m$ such that $F_{a_{1}} \cap$ $F_{a_{2}} \cap \cdots \cap F_{a_{l}}=\{0\}$.

Remark. The proof of Lemma (2.8) follows from two observations. We note first that for each finite subset $\left\{a_{1}, a_{2}, \cdots, a_{l}\right\}$ of $K, F_{a_{1}} \cap F_{a_{2}} \cap \cdots \cap F_{a_{l}}$ is a vector space. Secondly, if $F_{a_{1}} \cap F_{a_{2}} \cap \ldots \cap F_{a_{l}} \neq\{0\}$, then because $K$ is irreducible, we can find $a_{l+1}$ in $K$ for which $F_{a_{1}} \cap F_{a_{2}} \cap \cdots \cap F_{a_{l+1}}$ is a proper subspace of $F_{a_{1}} \cap F_{a_{2}} \cap \cdots \cap F_{a_{l}}$. Another approach would be to take the set of vectors which a given member of $K$ moves and try to enlarge this set. This does not appear to work, however, because the set of such vectors is not a vector space.

(2.9) Definition. Let the $F$-index of $K$ be the smallest positive integer $l$ for which there exist elements $a_{1}, a_{2}, \cdots, a_{l}$ in $K$ with the property that $F_{a_{1}} \cap$ $F_{a_{2}} \cap \cdots \cap F_{a_{l}}=\{0\}$.

Remark. The notion of $F$-index allows us to classify irreducible groups of $m \times m$ unitary matrices according to the number of distinct group elements necessary to generate a sequence $\left\{c_{v}\right\}$ with the property that $\left\langle\left\{c_{v}\right\}\right\rangle=0$. By Lemma (2.8), the $F$-index of an irreducible group of $m \times m$ unitary matrices exists and is bounded above by $m$. When there exists a matrix in $K$ with no eigenvalue 1 , then $K$ is of $F$-index 1. This is the case, for example, when $K$ is a nontrivial subgroup of the circle group or the group $U(m)$. Let $\rho$ denote the 3 -dimensional irreducible unitary representation of $A_{4}$, the altemating group on 4 elements, see $[7$, p. 49]. It is a straightforward but somewhat tedious matter to verify that the irreducible group of $3 \times 3$ unitary matrices given by $\rho\left(A_{4}\right)$ (with respect to some basis) is an example of a group of $F$-index 2 .

We can easily find a sequence $\left\{c_{v}\right\}$ in an irreducible group of $m \times m$ unitary matrices $K$ of $F$-index 1 such that $\langle\{c v\}\rangle=0$. Indeed, if $K$ is of $F$-index 1 , there exists a matrix $a \in K$ such that $F_{a}=\{0\}$. Then $\left\langle\left\{a^{v}\right\}\right\rangle=0$. Since every element of an irreducible group of $m \times m$ unitary matrices of $F$-index 2 or more has 1 as an eigenvalue, no sequence of powers of a single element will average to 0 . We now construct a sequence $\left\{c_{v}\right\}$ in an irreducible group of $m \times m$ unitary matrices of 
$F$-index $l, 1 \leq l \leq m$, with the property that $\left\langle\left\{c_{v}\right\}\right\rangle=0$.

(2.10) Suppose that the $F$-index of $K$ is some positive integer $l, 1 \leq l \leq m$. By Lemma (2.8) there exist elements $a_{1}, a_{2}, \cdots, a_{l}$ in $K$ with $\{0\}$ as the common fixed subspace. Using construction (2.1) we obtain a sequence $\left\{c_{v}\right\}$ in $K$ where the generating sequences $\{c(k, v)\}, k=1,2,3, \ldots$, are given by $\{c(k, v)\}=\left\{a_{j}^{v}\right\}$ and $k \equiv j(\bmod l), 1 \leq j \leq l$.

We illustrate the construction of $\left\{c_{v}\right\}$ in $\rho\left(A_{4}\right)$. It is easy to verify that $F_{\rho(123))} \cap F_{\rho((12)(34))}=\{0\}$. Let $\{c(k, v)\}=\left\{\rho((123))^{v}\right\}$ when $k$ is even and let $\{c(k, v)\}=\left\{\rho((12)(34))^{\nu}\right\}$ when $k$ is odd. Now construct $\left\{c v_{v}\right\}$ according to (2.1).

We observe that when $K$ is of $F$-index 1 the sequence $\left.\{c\}_{v}\right\}$ is slightly more complicated than the obvious sequence $\left\{a^{\nu}\right\}$ of powers of an appropriate element $a$ in $K$ such that $F_{a}=\{0\}$; however, the sequence $\left\{c_{v}\right\}$ still has only one generator.

We shall prove that the sequence $\left\{c_{v}\right\}$ in an irreducible group $K$ of $m \times m$ unitary matrices of $F$-index $l$ has the property that $\left\langle\left\{c_{v}\right\}\right\rangle=0$. The technique involves proving that the average of $\left\{c_{v}\right\}$ over all $n$th stages and $n$th stage-like blocks is suitably small for all sufficiently large $n$. The average of the sequence $\left\{c_{v}\right\}$ over an $n$th stage is essentially a product of projections $P_{l} P_{l-1} \cdots P_{1}$ raised to a power dependent on $n$. The sequence $\left\{c_{v}\right\}$ is constructed so that the projections $P_{l}, P_{l-1}, \cdots, P_{1}$ have no nonzero common fixed subspace. This implies that the operator $P_{l} P_{l-1} \cdots P_{1}$ has norm $\delta$ where $0 \leq \delta<1$. We then make the norm of the average over an $n$th stage of $\left\{c_{\nu}\right\}$ suitably small by making $n$ sufficiently large.

The following theorem is a slight modification of the ergodic theorem as stated in Halmos [4].

(2.11) Theorem [4]. If $U$ is an isometry on a finite dimensional inner product space and if $M$ is the space of all solutions of $U x=x$, then the sequence defined by $V_{n}=n^{-1}\left(U+U^{2}+\cdots+U^{n}\right)$ converges as $n \rightarrow+\infty$ to the perpendicular projection of $M$.

(2.12) Notation. Let $P_{i}(n)=n^{-1} \Sigma_{v=1}^{n} a_{i}^{v}$ and let $P_{i}=\lim _{n \rightarrow+\infty} P_{i}(n), i=1$, $2, \cdots, l$. We recall that the elements $a_{1}, a_{2}, \cdots, a_{l}$ of $K$ generate the sequence $\left\{c_{v}\right\}$.

By Theorem (2.11), $P_{i}$ exists for each $i$ and is a projection of $C^{m}$ onto $F_{a_{i}}{ }^{*}$

(2.13) Lemma. Let the F-index of $K$ be $l$ and let the elements $a_{1}, a_{2}, \ldots$, $a_{l}$ of $K$ bave $\{0\}$ as their common fixed subspace. Then there exists $\alpha, 0 \leq \alpha<1$, such that $\left\|P_{l} P_{l-1} \cdots P_{1}\right\| \leq a$.

Proof. The lemma is obvious when $l=1$. If $l \geq 2$ then we have $\| P_{l} P_{l-1} \cdots$ $P_{1}\|=\sup \| P_{l} \ldots P_{1} x \|$ where the supremum is taken over all $x,\|x\|=1$. It follows that $\left\|P_{l} \cdots P_{1}\right\|=\sup \left\|P_{l} \cdots P_{2} x\right\|$ where the supremum is taken over all 
$x \in F_{a_{1}},\|x\|=1$. We continue until we have $\left\|P_{l} \cdots P_{1}\right\|=\sup \left\|P_{l} x\right\|$ where the supremum is taken over all $x \in F_{a_{1}} \cap \ldots \cap F_{a_{l-1}}$ of norm 1. Let $x \in F_{a_{1}} \cap \ldots \cap$ $F_{a_{l}}$ and suppose $\|x\|=1$. Then $x=s+t$ where $s \in F_{a_{l}}$ and $t \in F_{a_{l}}^{\perp}$ so that $\left\|P_{l} x\right\|=\|s\|$. It is a straightforward matter to show that for all $y \in F_{a_{l}}$ such that $\|y\|=1$, there exists $a, 0 \leq a<1$, such that $|(x, y)|<a$. It follows that $|(x, s)| \leq a\|s\|$. Furthermore, $\|s\|^{2}=|(s+t, s)|=|(x, s)|$. Thus $\|s\| \leq a$. Therefore $\left\|P_{l} \ldots P_{1}\right\| \leq a<1$.

The next lemma states that the sequence $\left\{c_{v}\right\}$ constructed in $K$ according to (2.10) has the property that averages over all complete $n$th stages for all sufficiently large $n$ are small.

(2.14) Lemma. Let the F-index of $K$ be $l$ and let the elements $a_{1}, a_{2}, \ldots$, $a_{l}$ of $K$ bave $\{0\}$ as their common fixed subspace. Let $\left\{c_{v}\right\}$ be the sequence constructed in $K$ according to (2.10). Given $\epsilon>0$ there exists $M$ so that for all $n>M$,

$$
\left\|\frac{1}{n^{n}} \sum_{c_{\nu} \in C_{n}} c_{v}\right\|<\epsilon / 4 .
$$

Proof. By Lemma (2.13), $\left\|P_{l} \cdots P_{1}\right\|=\delta$ for some $\delta, 0 \leq \delta<1$. For each $i$, $1 \leq i \leq l,\left\|P_{i}(n)\right\| \leq 1$ for each positive integer $n$. It follows that there exists an integer $M_{1}$ so that for all $n>M_{1},\left\|P_{1} \ldots P_{1}-P_{l}(n) \ldots P_{1}(n)\right\|<(1-\delta) / 2$. Therefore $\left\|P_{l}(n) \ldots P_{1}(n)\right\|<(1+\delta) / 2$. Let $\epsilon>0$ be given. Since $(1+\delta) / 2<1$, there exists $M_{2}$ so that for all $n>M_{2},((1+\delta) / 2)^{(n-1) / l}<\epsilon / 4$. Let $M=$ $\max \left\{M_{1}, M_{2}\right\}$ and $n>M$. Then, for some integer $k, 1 \leq k \leq l, n \equiv k(\bmod l)$, we have

$$
\begin{aligned}
\left\|\frac{1}{n^{n}} \sum_{c_{\nu} \in C_{n}} c_{\nu}\right\| & -\left\|\frac{1}{n^{n}} \sum_{i=1}^{n} \sum_{t_{i}=1}^{n}\left(a_{k}^{t} \cdots a_{1}^{t n-k+1}\right)\left(a_{l}^{t} l^{n-k} \cdots a_{l}^{t n-k-l+1}\right) \cdots\left(a_{l}^{t} l \cdots a_{1}^{t}\right)\right\| \\
& -\left\|P_{k}(n) \cdots P_{1}(n)\left(P_{l}(n) \cdots P_{1}(n)\right)^{(n-k)^{\prime} l}\right\| \\
& <\epsilon / 4 .
\end{aligned}
$$

The lemma which follows states that the sequence $\left\{c_{v}\right\}$ as constructed in $K$ according to $(2.10)$ has the property that averages over all $n$th stage-like blocks for all sufficiently large $n$ are small.

(2.15) Lemma. Let $\left\{c_{v}\right\}$ and $M$ be as in Lemma (2.14). Let $n$ be a fixed integer greater than M. For each positive integer q,

$$
\left\|\frac{1}{(n+q)^{n}} \sum_{c_{\nu} \in B(i, q, n)} c_{v}\right\|<\epsilon / 4
$$

for eacb $i, 1 \leq i \leq(n+q)^{n}$. 
Proof. For some integer $k, 1 \leq k \leq l, n \equiv k(\bmod l)$. Let $q$ be any positive integer and suppose $n+q \equiv s(\bmod l), 1 \leq s \leq l$. For any $i, 1 \leq i \leq(n+q)^{q}$,

$$
\begin{aligned}
\left\|\frac{1}{(n+q)^{n}} \sum_{c_{v} \in B(i, q, n)} c_{v}\right\|=\| \frac{1}{(n+q)^{n}} \sum_{j=1}^{n} \sum_{t_{j}=1}^{n+q}\left(a_{s}^{t} n+q \ldots a_{1}^{t+q-s+1}\right) \\
\cdot\left(a_{l}^{t}{ }^{t+q-s} \ldots a_{1}^{t}+q-s-l\right) \cdots\left(a_{l}^{t} \cdots a_{1}^{t}\right) \| \\
\leq\left\|P_{k}(n+q) \cdots P_{1}(n+q)\right\|\left\|P_{l}(n+q) \cdots P_{1}(n+q)\right\|^{(n-k) / l} .
\end{aligned}
$$

Since $n>M,\left\|P_{l}(n+q) \ldots P_{1}(n+q)\right\|<(1+\delta) / 2$. Therefore,

$$
\left\|\frac{1}{(n+q)^{n}} \sum_{c_{v} \in B(i, q, n)} c_{v}\right\|<((1+\delta) / 2)^{(n-1) / l}<\epsilon / 4 .
$$

We now extend the notion of $F$-index to finite dimensional irreducible unitary representations of a locally compact group.

(2.16) Definition. Let $\rho \in R(G)$. The $F$-index of $\rho$ is the $F$-index of $\rho(G)$.

(2.17) Notation. Let $\Phi_{j}=\{\rho \in R(G) \mid F$-index of $\rho$ is $j\}, j=1,2,3, \ldots$ Let $\Phi_{0}$ be the subset of $R(G)$ containing only the trivial representation.

Remark. If $G$ is a locally compact abelian group, $\Phi_{0}$ and $\Phi_{1}$ exhaust $R(G)$.

We now state and prove the final result of this section.

(2.18) Lemma. Let $G$ be a locally compact group and $H$ a countable subgroup with the property that each nontrivial representation $\rho$ of some subset $S \subset R(G)$ is nontrivial and irreducible as a representation of $H$. Let $\rho$ be a nontrivial member of $S$ of dimension $m$. Then there exists a sequence $\left\{b_{v}\right\}$ in $H$ (dependent on $\rho)$ with at most $m$ generators such that $\left\langle\rho,\left\{b_{v}\right\}\right\rangle=0$.

Proof. Since $\rho$ is a nontrivial member of $S$ of dimension $m$, it follows that $\rho(H)$ is a nontrivial irreducible group of $m \times m$ unitary matrices. The $F$-index of $\rho$ is $l$ where $1 \leq l \leq m$. Therefore there exist elements $a_{1}, a_{2}, \cdots, a_{l}$ in $\rho(H)$ with $\{0\}$ as their common fixed subspace. We use construction (2.1) to obtain $\left\{c_{v}\right\}$ in $\rho(H)$ where the generating sequences $\{c(k, v)\}, k=1,2,3, \ldots$, are given by $\{c(k, v)\}=\left\{a_{j}^{v}\right\}$ where $k \equiv j(\bmod l), 1 \leq j \leq l$. From Lemmas (2.6), (2.14), and (2.15) we see that $\left\langle\left\{c_{v}\right\}\right\rangle=0$. Let $k_{i}$ be an element of $\rho^{-1}\left(a_{i}\right), i=1,2, \cdots, l$. We construct a sequence $\left\{b_{v}\right\}$ in $H$ generated by $k_{i}$ with the property that $\left\langle\rho,\left\{b_{v}\right\}\right\rangle=0$.

Remark. We are still far from the proof of the key lemma in the lemma just proved. The sequence $\left\{b_{v}\right\}$ in Lemma (2.18) averages out a single representation to 0 , while the key lemma asserts under the same hypotheses as Lemma (2.18) the existence of a single universal sequence $\left\{b_{v}\right\}$ in $H$ which averages all nontrivial representations $\rho$ in $S$ to 0 . The assumption of the countability of $H$ is not necessary in Lemma (2.18) but becomes crucial in the construction of the universal sequence in $\$ 3$. 
3. The main theorem. In this section we complete the proof of the key lemma. Using this lemma, we prove that a locally compact group possesses a u.d. sequence if and only if it is $K$-separable.

(3.1) Lemma. Let $G$ be a locally compact group. Let $H$ be a countable subgroup with the property that each nontrivial $\rho$ in some subset $S$ of $R(G)$ is nontrivial and irreducible as a representation of $H$. Then for eacb integer $j \geq 1$ there exists a sequence $\left\{c_{v}\right\}$ in $H$ sucb that $\left\langle\rho,\left\{c_{v}\right\}\right\rangle=0$ for each $\rho \in S \cap \Phi_{j}$.

Proof. If $S \cap \Phi_{j}=\varnothing$, then let $\left\{c_{v}\right\}$ be the sequence whose terms are the identity $e$ of $H$. If $\rho \in S \cap \Phi_{j}$, then, by Lemma (2.8) there exists a $j$-tuple of elements of $H,\left(b_{1}, b_{2}, \cdots, b_{j}\right)$, with the property that $F_{\rho\left(b_{1}\right)} \cap F_{\rho\left(b_{2}\right)} \cap \cdots \cap$ $F_{\rho\left(b_{j}\right)}=\{0\}$. Furthermore, by Lemma (2.18) the sequence $\left\{c_{v}\right\}$ generated according to $(2.10)$ from the sequences of powers $\left\{b_{1}^{n}\right\},\left\{b_{2}^{n}\right\}, \ldots,\left\{b_{j}^{n}\right\}$ has the property that $\left\langle\rho,\left\{c_{v}\right\}\right\rangle=0$.

Because $H$ is countable, there are at most countably many $j$-tuples of elements of $H$. Let $H_{0}$ be a set of $j$ tuples $\left\{p_{1}, p_{2}, \cdots, p_{k}, \cdots\right\}$ of elements of $H$ such that:

(i) For each $\rho$ in $S \cap \Phi_{j}$ there is a j-tuple $p_{k}$ of elements of $H$ in $H_{0}$ and a sequence $\{c(k, v)\}$ in $H$ generated by the components of $p_{k}$ with the property that $\langle\rho,\{c(k, v)\}\rangle=0$.

(ii) Each $j$-tuple $p_{k}$ in $H_{0}$ corresponds to a sequence $\{c(k, v)\}$ in $H$ generated by the components of $p_{k}$ and $\langle\rho,\{c(k, v)\}\rangle=0$ for some $\rho$ in $S \cap \Phi_{j}$.

We now have a collection of sequences $\{c(1, v)\},\{c(2, v)\}, \ldots,\{c(k, v)\}, \ldots$ corresponding to the elements $p_{k}, k=1,2, \ldots$ of $H_{0}$. Using the sequences $\{c(1, v)\},\{c(2, v)\}, \ldots,\{c(k, v)\}, \ldots$ as generators, we construct a new sequence $\left\{c_{v}\right\}$ in $H$ and hence in $G$ according to (2.1). The construction we recall is in stages. The first stage is $c_{11}$. The second stage is $c(2,1) c(1,1), c(2,1) c(1,2)$, $c(2,2) c(1,1), c(2,2) c(1,2)$. The $n$th state is all terms of the form $c\left(n, t_{n}\right) c\left(n-1, t_{n-1}\right) \cdots c\left(1, t_{1}\right)$ where $t_{i}$ runs from 1 to $n, i=1,2, \cdots, n$. For more details refer to (2.1). If $H_{0}$ happens to be finite, say $H_{0}=\left\{p_{1}, p_{2}, \cdots, p_{k}\right\}$, let $c(l, v)$ be the identity $e$ of $H$ for all positive integers $v$ and all positive integers $l, l>k$. This completes the construction of the sequence $\left\{c_{v}\right\}$.

Let $\rho$ be an arbitrary element of $S \cap \Phi_{j}$. Let $p_{k}=\left(b\left(i_{1}\right), b\left(i_{2}\right), \ldots, b\left(i_{j}\right)\right)$ be the first $j$ tuple in $H_{0}$ for which $F_{\rho\left(b\left(i_{1}\right)\right)} \cap F_{\rho\left(b\left(i_{2}\right)\right)} \cap \cdots \cap F_{\rho\left(b\left(i_{j}\right)\right)}=\{0\}$. In the construction of $\left\{c_{v}\right\}$ we use the sequence $\{c(k, v)\}$ in generating $\left\{c_{v}\right\}$. The sequence $\{c(k, v)\}$ we recall is generated by the sequences of powers of $b\left(i_{1}\right)$, $b\left(i_{2}\right), \cdots, b\left(i_{j}\right)$. In addition, $\{c(k, v)\}$ has the property that for any $\epsilon>0$ there exists $N_{0}$ such that for all $n>N_{0}$, 


$$
\left\|\frac{1}{n} \sum_{\nu=1}^{n} \rho(d(k, v))\right\|<\epsilon .
$$

Set $N=\max \left\{\Sigma_{i=1}^{N_{0}} i^{i}, \Sigma_{i=1}^{k} i^{i}\right\}$ and let $n>N$. We have

$\left\|\frac{1}{n^{n}} \sum_{c_{v} \in C_{n}} \rho\left(c_{\nu}\right)\right\|=\| \frac{1}{n^{n}} \sum_{i=1}^{n} \sum_{t_{i}=1}^{n} \rho\left(d\left(n, t_{n}\right) d\left(n-1, t_{n-1}\right) \cdots\right.$

$$
\left.c\left(k, t_{k}\right) \cdots c\left(1, t_{1}\right)\right)
$$

$$
\leq\left\|\frac{1}{n^{n-k}} \sum_{i=k+1}^{n} \sum_{t_{i}=1}^{n} \rho\left(d\left(n, t_{n}\right) \cdots c\left(k+1, t_{k+1}\right)\right)\right\|
$$$$
\text { - }\left\|\frac{1}{n} \sum_{t_{k}=1}^{n} \rho\left(d\left(k, t_{k}\right)\right)\right\|\left\|\frac{1}{n^{k-1}} \sum_{i=1}^{k-1} \sum_{t_{i}=1}^{n} \rho\left(d\left(k-1, t_{k-1}\right) \ldots d\left(1, t_{1}\right)\right)\right\| \text {. }
$$

The matrices $\rho\left(c\left(n, t_{n}\right) \cdots c\left(k+1, t_{k+1}\right)\right)$ and $\rho\left(c\left(k-1, t_{k-1}\right) \cdots c\left(1, t_{1}\right)\right)$ are unitary for all permissible values of $t_{1}, t_{2}, \cdots, t_{k-1}, t_{k+1}, \cdots, t_{n}$. Therefore

(i)

$$
\left\|\frac{1}{n^{n}} \sum_{c_{v} \in C_{n}} \rho\left(c_{v}\right)\right\|<1 \cdot \epsilon \cdot 1 .
$$

We recall that $B(i, q, n)$ is an $n$th stage-like block of $\left\{c_{v}\right\}$, see (2.1). Assume $n>N$ and let $q$ be any positive integer. Let $1 \leq i \leq(n+q)^{q}$. Then

$\left\|\frac{1}{(n+q)^{n}} \sum_{c_{v} \in B(i, q, n)} \rho\left(c_{v}\right)\right\|=\left\|\frac{1}{(n+q)^{n}} \sum_{l=1}^{n} \sum_{t_{l}=1}^{n+q} \rho\left(c\left(n+q_{0} t_{n+q}\right) \cdots d\left(1, t_{1}\right)\right)\right\|$

$$
\begin{aligned}
\leq & \left\|\rho\left(c\left(n+q, t_{n+q}\right) \cdots c\left(n+1, t_{n+1}\right)\right)\right\| \\
& \cdot\left\|\frac{1}{(n+q)^{n-k}} \sum_{l=k+1}^{n} \sum_{t_{l}=1}^{n+q} \rho\left(c\left(n, t_{n}\right) \cdots c\left(k+1, t_{k+1}\right)\right)\right\| \\
& \cdot\left\|\frac{1}{n+q} \sum_{t_{k}=1}^{n+q} \rho\left(c\left(k, t_{k}\right)\right)\right\| \\
& \cdot\left\|\frac{1}{(n+q)^{k-1}} \sum_{l=1}^{k-1} \sum_{t_{l}=1}^{n+q} \rho\left(c\left(k-1, t_{k-1}\right) \cdots c\left(1, t_{1}\right)\right)\right\| .
\end{aligned}
$$

The matrices $\rho\left(c\left(n+q, t_{n+q}\right) \cdots c\left(n+1, t_{n+1}\right)\right), \rho\left(c\left(n, t_{n}\right) \cdots c\left(k+1, t_{k+1}\right)\right)$, and $\rho\left(c\left(k-1, t_{k-1}\right) \cdots c\left(1, t_{1}\right)\right)$ are unitary for all permissible values of $t_{n+q}$, $\cdots, t_{k+1}, t_{k-1}, \cdots, t_{1}$. Therefore, 
(ii) $\left\|\frac{1}{(n+q)^{n}} \sum_{c_{\nu} \in B(i, q, n)} \rho\left(c_{v}\right)\right\| \leq 1 \cdot 1 \cdot\left\|\frac{1}{n+q} \sum_{t_{k}=1}^{n+q} \rho\left(c\left(k, t_{k}\right)\right)\right\| \cdot 1<\epsilon$.

By inequalities (i) and (ii) it follows that the sequence $\left\{\rho\left(c_{\nu}\right)\right\}$ satisfies the hypotheses of Lemma (2.6). By Lemma (2.6), $\left\langle\rho,\left\{c_{v}\right\}\right\rangle=0$.

Remark. The countability of $H$ is crucial in the construction of the sequence $\left\{c_{v}\right\}$; however, the number of finite dimensional irreducible unitary representations of $F$-index $j$ is unrestricted. For example, let $G$ be $R$, the reals in the usual topology. The rationals form a countable subgroup with the property that each nontrivial character of $R$ is nontrivial as a character on the rationals. Using the construction just given, we can find a sequence of real numbers which averages all nontrivial characters on $R$ to 0 . We observe that $R$ has uncountably many nontrivial characters; that is, $R$ has uncountably many finite dimensional irreducible unitary representations of $F$-index 1 .

We now state and prove our key lemma.

(3.2) Lemma. Let $G$ be a locally compact group and $H$ a countable subgroup. Suppose that for each nontrivial $\rho$ in some subset $S$ of $R(G), \rho$ is nontrivial and irreducible as a representation of $H$. Then there exists a sequence $\left\{c_{v}\right\}$ in $H$ sucb that $\left\langle\rho,\left\{c_{v}\right\}\right\rangle=0$ for all nontrivial $\rho$ in $S$.

Proof. By Lemma (3.1) there exist countably many sequences $\{c(k, v)\}, k=1$, $2,3, \cdots$, in $H$ with the property that $\langle\rho,\{c(k, v)\}\rangle=0$ for every $\rho$ in $S \cap \Phi_{k}$. If $S \cap \Phi_{k}=\varnothing$, then let $c(k, v)$ be the identity of $H$ for all possible values of $v$. Using (2.1), we construct a new sequence $\left\{c_{v}\right\}$ in stages. We use the sequences $\{c(k, v)\}, k=1,2,3, \cdots$, from Lemma (3.1) as generators. The first stage is $c(1,1)$. The second stage is $c(2,1) c(1,1), c(2,1) c(1,2), c(2,2) c(1,1)$, $c(2,2) c(1,2)$. The $n$th stage is all terms of the form $c\left(n, t_{n}\right) c\left(n-1, t_{n-1}\right) \cdots$ $c\left(1, t_{1}\right)$ where $t_{i}$ runs from 1 to $n, i=1,2, \ldots, n$. For more details see (2.1).

Let $\rho$ be a nontrivial member of $S$. It follows that $\rho \in S \cap \Phi_{j}$ for some integer $j \geq 1$. By the construction of $\left\{c_{v}\right\}$, one of the generating sequences $\{c(j, v)\}$ for $\left\{c_{v}\right\}$ has the property that there exists $N_{0}$ such that for all $n>N_{0}$,

$$
\left\|\frac{1}{n} \sum_{v=1}^{n} \rho(c(j, v))\right\|<\epsilon .
$$

Set $N=\max \left\{\Sigma_{i=1}^{N_{0}} i^{i}, \Sigma_{i=1}^{j} i^{i}\right\}$ and let $n>N$. Then

$$
\left\|\frac{1}{n^{n}} \sum_{c_{v} \in C_{n}} \rho\left(c_{v}\right)\right\|<\epsilon .
$$

Also, for each $n>N$, it follows that for each positive integer $q$, 


$$
\left\|\frac{1}{(n+q)^{n}} \sum_{c_{v} \in B(i, q, n)} \rho\left(c_{\nu}\right)\right\|<\epsilon
$$

for each $i, 1 \leq i \leq(n+q)^{q}$. We omit the proof of the two preceding inequalities since it is somewhat similar to the proof of inequalities (i) and (ii) of Lemma (3.1). By Lemma (2.6), $\left\langle\rho,\left\{c_{v}\right\}\right\rangle=0$.

The remarks which follow allow us to generalize Lemma (3.2). As a special case of this generalization, we obtain a reformulation of Lemma (3.2) which is very similar to the analogue [1, Lemma 2.1$]$ in the abelian case.

Remark. It is a straightforward matter to show that a finite dimensional unitary representation $\rho$ of a locally compact group $G$ has no nonzero fixed vector if and only if for each m.a.i. function $f$ of $\rho$ (independent of basis) there exists $g \in G$ such that $f(g) \neq 1$.

Remark. We observe that Lemma (2.8) holds under the weaker hypothesis that $K$ is a group of $m \times m$ unitary matrices (not necessarily irreducible) which fixes no nonzero vector in $C^{m}$. We are still assured of the existence of a set $\left\{a_{1}, a_{2}\right.$, $\left.\cdots, a_{l}\right\}$ in $K, 1 \leq l \leq m$, such that $F_{a_{1}} \cap F_{a_{2}} \cap \cdots \cap F_{a_{l}}=\{0\}$. Thus we may extend the notion of $F$-index to a group of $m \times m$ unitary matrices which has no nonzero fixed vector. Furthermore, we may define, in the natural way, the $F$-index of any $m$-dimensional unitary representation of a locally compact group which fixes no nonzero vector in $C^{m}$.

Remark. Let $G$ be a locally compact group and let $\rho$ be a finite dimensional (not necessarily irreducible) unitary representation of $G$. Then any nontrivial m.a.i. function $f$ of $\rho$ is orthogonal to 1 . Indeed, we may consider any nontrivial m.a.i. function $f$ as a m.a.i. function of the representation $\rho$ on the Bohr compactification of $G$. We may decompose $\rho$ into a direct sum of irreducible unitary representations on the Bohr compactification, see [6, Theorem 21.40]. The function $f$ is then a finite linear combination of m.a.i. functions from nontrivial irreducible unitary representations of the Bohr compactification and hence is orthogonal to 1 .

The preceding remarks enable us to generalize Lemma (3.2) as follows.

(3.3) Lemma. Let $G$ be a locally compact group and $S$ a subset of the set of finite dimensional (not necessarily irreducible) unitary representations of G. Let $H$ be a countable subgroup with the property that for eacb nontrivial m.a.i. function $f$ from a representation $\rho$ in $S$ there exists $b$ in $H$ such that $f(b) \neq 1$. Then there exists a sequence $\left\{c_{v}\right\} \subset H$ such that $\left\langle f,\left\{c_{v}\right\}\right\rangle=0$ for eacb nontrivial m.a.i. function from any representation $\rho$ in $S$.

As a special case of Lemma (3.3) we have the following. 
(3.4) Lemma. Let $G$ be a locally compact group and $H$ a countable subgroup of $G$. Let $S$ be a subset of $R(G)$ and suppose that for eacb nontrivial m.a.i. function $f$ from a representation in $S$ there exists $b$ in $H$ sucb tbat $f(b) \neq 1$. Then there exists a sequence $\left\{c_{\nu}\right\}$ in $H$ sucb that $\left\langle\rho,\left\{c_{v}\right\}\right\rangle=0$ for all nontrivial $\rho$ in $H$.

We now state the analogue to Lemma (3.2) in the abelian case. We note the similarity between [1, Lemma 2.1] and our Lemma (3.4). It is also of interest to compare a result of Hlawka concerning sequences on compact groups [8, Theorem 7] and Lemma (3.4).

(3.5) Lemma [1, Lemma 2.1]. Let $G$ be a locally compact abelian group and $S$ a subset of the character group $G^{\wedge}$. Let $H$ be a countable subgroup of $G$ witb the property that for each nontrivial character $\rho$ in $S$ there is an element $b$ in $H$ sucb that $\rho(b) \neq 1$. Then there is a sequence $\left\{b_{\nu}\right\}$ of elements of $H$ sucb tbat $\left\langle\rho,\left\{b_{v}\right\}\right\rangle=0$ for all nontrivial $\rho$ in $S$.

We now characterize in terms of $K$-separability those locally compact groups which admit uniformly distributed sequences. It would be interesting, of course, to have a characterization in terms of group structure. In the abelian case, for example, in [1, Theorem 3] Berg, Rajagopalan, and Rubel relate the existence of u.d. sequences to the cardinality of the set of periodic characters.

(3.6) Theorem. A locally compact group $G$ possesses a u.d. sequence if and only if it is K-separable.

Proof. Suppose $G$ has a u.d. sequence $\left\{g_{v}\right\}$. Let $H$ be any closed normal subgroup of compact index in $G$. Then $\left\{\phi_{H}\left(g_{v}\right)\right\}$ is u.d. in $G / H$, a compact group. But a sequence which is u.d. in a compact group is dense so $G$ is $K$-separable.

Assume that $G$ is $K$-separable. Then $G$ possesses a $K$-dense sequence $\left\{g_{v}\right\}$. Let $H$ be the countable subgroup of $G$ generated by $\left\{g_{v}\right\}$. Let $\rho$ be a nontrivial member of $P$, the irreducible periodic unitary representations of $G$. We may consider $\rho$ as an irreducible unitary representation of $G / W$ for some closed normal subgroup $W$ of compact index in $G$. Since $\rho(G)=\rho\left(\phi_{W}(G)\right), \rho(H)=\rho\left(\phi_{W}(H)\right)$. Furthermore, $\rho$ is irreducible as a representation of $\phi_{W}(G)$. It follows from the continuity of $\rho$ and the fact that $\phi_{W}(H)$ is dense in $\phi_{W}(G)$ that $\rho$ is irreducible as a representation of $\phi_{W}(H)$. Therefore $\rho$ is irreducible as a representation of $H$. By similar reasoning, it follows that $\dot{\rho}$ is al so nontrivial as a representation of $\boldsymbol{H}$. In Lemma (3.2), let $S=P$. Then there exists a sequence $\left\{b_{v}\right\}$ in $H$ such that $\left\langle\rho,\left\{b_{\nu}\right\}\right\rangle=0$ for every nontrivial $\rho$ in $P$. By the Weyl criterion for locally compact groups, $\left\{b_{v}\right\}$ is u.d. in $G$.

Acknowledgment. Thanks are due to Professor I. D. Berg of the University of Illinois for suggesting this line of research and for his helpful advice and comments. 


\section{BIBLIOGRAPHY}

1. I. D. Berg, M. Rajagopalan and L. A. Rubel, Uniform distribution on locally compact Abelian groups, Trans. Amer. Math. Soc. 133 (1968), 435-446. MR 37 \#3279.

2. I. D. Berg and L. A. Rubel, Densities on locally compact Abelian groups, Ann. Inst. Fourier (Grenoble) 19 (1969), fasc. 1, 81-107. MR 40 \#3178.

3. B. Eckmann, Über monothetische Gruppen, Comment. Math. Helv. 16 (1944), 249-263. MR 6, 146.

4. P. R. Halmos, Finite-dimensional vector spaces, 2nd ed., University Series in Undergraduate Math., Van Nostrand, Princeton, N. J., 1958. MR 19, 725.

5. S. Hartman, Remarks on equidistribution on noncompact groups, Compositio Math. 16 (1964), 66-71. MR 30 \#184.

6. E. Hewitt and K. Ross, Abstract harmonic analysis. Vol. I: Structure of topological groups. Integration theory, group representations, Die Grundlehren der math. Wissenschaften, Band 115, Academic Press, New York; Springer-Verlag, Berlin, 1963. MR 28 \#158.

7. - Abstract harmonic analysis. Vol. II: Structure and analysis for compact groups analysis on locally compact Abelian groups, Die Grundlehren der math. Wissenschaften, Band 152, Springer-Verlag, Berlin and New York, 1970. MR 41 \#7378.

8. E. Hlawka, Zur formalen Theorie der Gleichverteilung in kompacten Gruppen, Rend. Circ. Mat. Palermo Ser. (2) 4 (1955), 33-47. MR 17, 594.

9. L. A. Rubel, Uniform distribution in locally compact groups, Comment. Math. Helv. 39 (1965), 253-258. MR 31 \#3537.

10. H. Weyl, Uber die Gleichverteilung von Zahlen mod Eins, Math. Ann. 77 (1916), 313-352; Selecta H. Weyl (1956), 111.

805 CENTENNIAL DRIVE, CHAMPAIGN, ILLINOIS 61820 